\title{
An unexplored MBE growth mode reveals new properties of superconducting $\mathrm{NbN}$
}

\author{
John Wright, ${ }^{1}$ Celesta Chang, ${ }^{2}$ Dacen Waters, ${ }^{3}$ Felix Lüpke, ${ }^{3}$ Lucy Raymond, ${ }^{4}$ Rosalyn Koscica, ${ }^{4}$ Guru \\ Khalsa, ${ }^{4}$ Randall Feenstra, ${ }^{3}$ David Muller, ${ }^{5,6}$ Huili G. Xing, ${ }^{1,7,8}$ and Debdeep Jena ${ }^{1,7,8}$ \\ ${ }^{1)}$ Department of Materials Science and Engineering, Cornell University, Ithaca, New York 14853, \\ USA. \\ 2) Department of Physics, Cornell University, Ithaca, New York, 14853, USA \\ ${ }^{3)}$ Department of Physics, Carnegie Mellon University, Pittsburgh, Pennsylvania 15213, \\ USA \\ 4) Department of Materials Science and Engineering, Cornell University, Ithaca, New York, 14853, \\ USA \\ 5) Department of Applied and Engineering Physics, Cornell University, Ithaca, New York, 14853, \\ USA \\ ${ }^{6)}$ Kavli Institute for Nanoscale Science, Cornell University, Ithaca, New York, 14853, \\ USA \\ 7) School of Electrical and Computer Engineering, Cornell University, Ithaca, New York 14853, \\ USA. \\ ${ }^{8)}$ Kavli Institute for Nanoscale Science, Cornell University, Ithaca, New York 14853, USA.
}

\begin{abstract}
Accessing unexplored crystal growth conditions often reveal new regimes of physical behavior. In this work, performing molecular beam epitaxy growth of the technologically important superconductor $\mathrm{NbN}$ at temperatures greater than $1000^{\circ} \mathrm{C}$ reveals a growth mode that has not been accessed before. This mode results in persistent RHEED oscillations through the entire growth, resulting in atomically smooth surfaces, normal metal resistivities of $\sim 37 \mu \Omega$-cm. We find that the superconducting critical temperature depends strongly on growth temperature, and report a maximum superconducting critical temperature of $15.5 \mathrm{~K}$. Electron-microscopy studies reveal a rich range of crystalline phases that depend on the growth temperature and correlate to the physical properties. Surprisingly, a reversal of the sign of the Hall coefficient from n-type to p-type is observed as the $\mathrm{NbN}$ films are cooled, indicating an electronic structure that has not been observed before in this material. In addition to this observation, the crystallinity of the high-temperature epitaxial $\mathrm{NbN}$ allows for an ordered Abrikosov vortex lattice to be imaged for the first time in this superconductor.
\end{abstract}

\section{INTRODUCTION}

Superconductivity in NbN was discovered in $1941^{1}$. For several years it was was the material with the highest measured superconducting critical temperature at about $17 \mathrm{~K}$. Though materials with higher critical temperatures have since been discovered, $\mathrm{NbN}$ is still commonly used in superconducting electronics due in part to the relative ease of thin film deposition, its appreciable critical temperature, its compatibility with standard semiconductor fabrication methods. $\mathrm{NbN}$ thin films are used for a wide variety of superconductive electronic devices, including qubits ${ }^{2}$, superconducting nanowire single photon detectors (SNSPD) ${ }^{3}$ and single flux quantum circuits ${ }^{4}$. Most commonly, $\mathrm{NbN}$ thin films have been created with sputter deposition techniques ${ }^{5}$, though pulsed laser deposition ${ }^{6}$, atomic layer desposition ${ }^{7}$, chemical vapor deposition $^{8}$, and molecular beam epitaxy $(\mathrm{MBE})^{9,10}$. Sputter deposition has proven capable of producing $\mathrm{NbN}$ films with critical temperatures of $17 \mathrm{~K}$ and resistivities as low as $31 \mu \Omega$ $\mathrm{cm}$. Yet in applications where $\mathrm{NbN}$ films thinner than $10 \mathrm{~nm}$ are desired, film nucleation, coalescence, and epitaxy become increasingly important.

Recently there is growing interest in integrated heteroepitaxial thin film structures combining superconducting, metallic, insulating, and semiconducting materials to advance quantum computation and communication. For instance, allepitaxial Josephson junctions have been proposed as a potential method to eliminate the decoherence in qubits caused by amorphous barrier materials ${ }^{11}$. We hypothesize that if the precise and ultra-thin layer thicknesses, atomically sharp interfaces, and low defect density characteristic of III-N semiconductor heterostructures grown by MBE could include superconducting transition metal nitrides, novel devices could be realized ${ }^{12,13}$.

In this work, growth of $\mathrm{NbN}$ thin films by MBE at high temperatures is shown to offer advantages in film quality due to the realization of a novel two-dimensional growth mode not previously reported for this material grown by any method. We find a $2 \mathrm{D}$ growth mode of $\mathrm{NbN}$ for substrate temperatures at or above what is used in most MBE reactors. Films grown in these conditions exhibit positive room temperature Hall coefficients, Hall coefficient reversal as a function of temperature, and the formation of an ordered Abrikosov vortex lattice, properties not seen before in the metallic and superconducting phases of $\mathrm{NbN}$.

\section{METHODS}

\section{A. Thin Film Growth}

$\mathrm{NbN}$ thin films were grown on Si-face $6 \mathrm{H}-\mathrm{SiC}$ substrate by nitrogen plasma-assisted molecular beam epitaxy using a Veeco GENxplor MBE system in which the base pressure is below $10^{-10}$ Torr. $\mathrm{Nb}$ is supplied by an electron-beam evaporator, and $\mathrm{Nb}$ flux is measured using an electron impact energy spectroscopy (EIES) system. Film growth is monitored in situ using RHEED. The $6 \mathrm{H}-\mathrm{SiC}$ substrate was treated by the vendor with a $\mathrm{H}_{2}$ gas anneal to achieve an atomically terraced surface. Prior to loading into the reactor, substrates were 
(a)

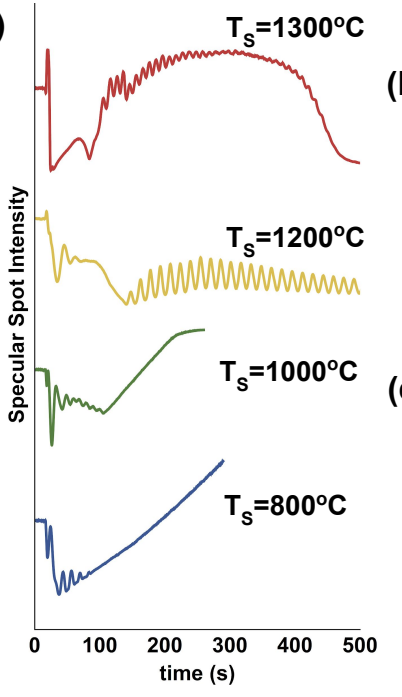

(b)
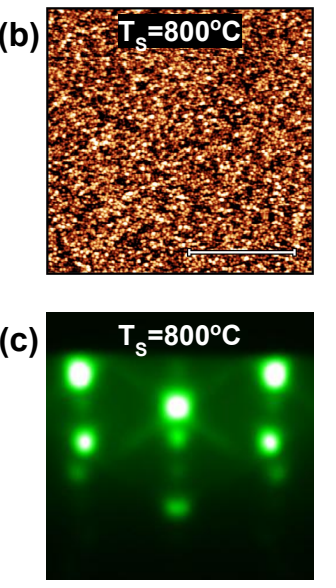
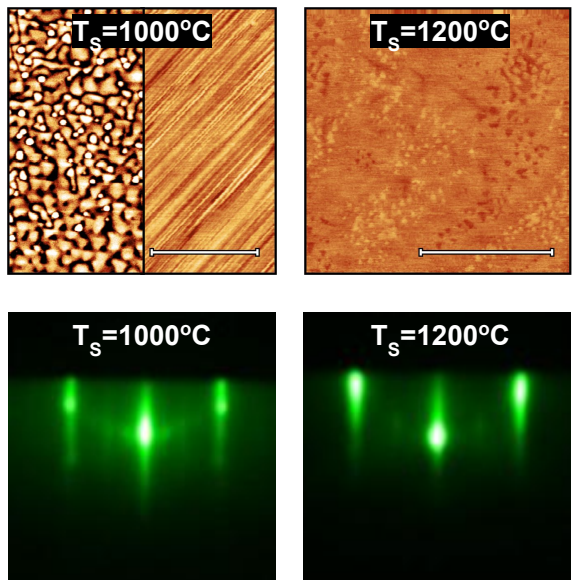
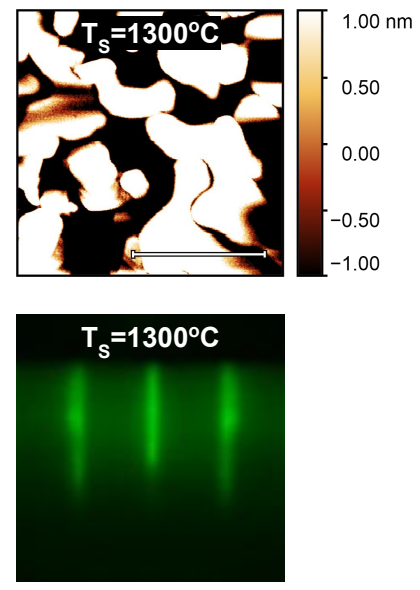

FIG. 1 : (a) In-situ RHEED specular spot peak intensity as a function of time throughout the first 500 s of growth of $\mathrm{NbN}$ films on $6 \mathrm{H}$ $\mathrm{SiC}$ substrate for different substrate temperatures. (b) Post-growth AFM surface height maps for NbN films grown at different substrate temperatures. The scale bars correspond to $400 \mathrm{~nm}$. (c) In-situ post-growth RHEED patterns.

rinsed with solvents followed by rinsing in piranha solution. Immediately prior to loading into the reactor chamber the substrate surface was rinsed with hydrofluoric acid, followed by deionized water to remove the surface native oxide layer. We attempted treating the surface using deposition and thermal desorption of $\mathrm{Ga}$ in the MBE reactor chamber to further remove the substrate oxidation layer, a practice which has been utilized for MBE growth of $\mathrm{AlN}$ on $6 \mathrm{H}-\mathrm{SiC}^{14}$. However, we found that this practice was correlated with an inability to observe evidence of a 2-dimensional $\mathrm{NbN}$ growth mode. A series of $\mathrm{NbN}$ samples all approximately $30 \mathrm{~nm}$ thick were grown using a range of substrate temperatures. A nitrogen flow rate of 1.95 SCCM and a plasma power of $200 \mathrm{~W}$ were used for all growths. The chamber pressure during growth obtained with these conditions was $1.7 \times 10^{-5}$ Torr; it was observed that the chamber pressure dropped by approximately $10 \%$ as the $\mathrm{Nb}$ flux is increased.

The active nitrogen flux for these plasma conditions was determined to be 4.4 atom $\cdot \mathrm{nm}^{-2} \cdot \mathrm{s}^{-1}$ by measuring the growth rate of $\mathrm{GaN}$ grown in nitrogen limited growth conditions in the same system. For all films presented in this study the flux of active nitrogen during growth exceeds the $\mathrm{Nb}$ flux. Growth rates varied slightly between samples, from approximately 0.16 to $0.22 \AA / s$. These growth rates correspond to a $\mathrm{Nb}$ flux between 0.76 and 1.0 atom $\cdot \mathrm{nm}^{-2} \cdot \mathrm{s}^{-1}$. Substrate temperatures during growth are measured by a thermocouple mounted behind the substrate and are varied between $800{ }^{\circ} \mathrm{C}$ and $1700^{\circ} \mathrm{C}$. We report the uncalibrated substrate thermocouple temperatures during growth despite our awareness that the substrate thermocouple is not always an accurate measurement of the true surface temperature during growth. However even for a constant substrate thermocouple reading the surface temperature varies during the growth of the $\mathrm{NbN}$ film due to increasing absorption of infrared thermal radiation by the high density of conduction electrons in the metallic $\mathrm{NbN}^{15}$.

\section{B. Crystal Structure and Electronic Properties}

Film surface morphology and roughness were characterized by atomic force microscopy using an Asylum Research Cypher ES system operated in tapping mode. In-situ phase characterization was performed using a RHEED system operated at $15 \mathrm{kV}$ and $1.5 \mathrm{~A}$. The structural phase and orientation of the films were analyzed using XRD coupled $2 \theta / \omega$ and RSM techniques with a Malvern Panalytical Empyrean diffractometer at $45 \mathrm{kV}, 40 \mathrm{~mA}$ with $\mathrm{Cu} \mathrm{K} \alpha 1$ radiation (1.54057 $\AA$ ). Film thickness and growth rate were determined using X-ray reflectivity (XRR). Crystal orientation and grain structure were investigated using a Tescan Mira3 SEM-EBSD system. The $\mathrm{Nb}: \mathrm{N}$ ratio was measured by RBS.

Further structural analysis was performed by preparing TEM cross-sectional samples using Helios G4-UX Focused Ion Beam (FIB). Prior to ion milling, carbon and platinum were deposited to protect the surface from potential ion-beam damage. The final milling was done at $5 \mathrm{kV}$ to reduce redeposition. HAADF-STEM imaging was performed using an aberration-corrected Titan Themis at an acceleration voltage of $300 \mathrm{keV}$.

Normal metal and superconducting properties were characterized using Van der Pauw resistivity and Hall effect measurements as a function of temperature in a Quantum Design PPMS system. The superconducting properties were further characterized with STM measurements at $4.7 \mathrm{~K}$.

\section{RESULTS}

\section{A. Epitaxial growth and structural properties}

The significant effect of temperature on the growth of the $\mathrm{NbN}$ thin films is first discussed. During growth of $\mathrm{NbN}$ on $6 \mathrm{H}-\mathrm{SiC}$, oscillations of the RHEED specular spot intensity are observed at nucleation, but typically persist only for a time that corresponds to the growth of several $\mathrm{NbN}$ layers (Fig. 
1(a)). As seen in Fig. 1(c), films grown at $800{ }^{\circ} \mathrm{C}$ exhibit spotty RHEED patterns during and after growth, indicating a 3-dimensional film surface. AFM investigation of the $\mathrm{NbN}$ films grown at $800^{\circ} \mathrm{C}$ (Fig. 1(b)) show a smooth surface composed of grains whose diameter on the surface is less than $50 \mathrm{~nm}$. Both the RHEED and AFM data for films grown at $800{ }^{\circ} \mathrm{C}$ are indicative of a 3-dimensional Volmer-Weber (island formation) growth mode.

Films grown at a substrate temperature of $1000{ }^{\circ} \mathrm{C}$ show oscillations of the RHEED specular spot brightness that persisted for slightly more than $100 \mathrm{~s}$ (Fig. 1(a)). The RHEED pattern of films grown at $1000{ }^{\circ} \mathrm{C}$ contains a combination of streaky and spotty features (Fig. 1(c)). The surfaces of these films possess some regions with triangular pyramidal grains indicative of 3-dimensional growth of a 111 oriented cubic film, while other regions possess parallel striped facets across the surface (Fig. 1(b)). The width of each stripe is approximately $10 \mathrm{~nm}$, and the height difference between peak and valley is approximately $0.5 \mathrm{~nm}$. The two morphologies are distributed randomly across the sample surface and can be distinguished through an optical microscope. Striped regions of different orientations meet forming $60^{\circ}$ angles. This striped morphology appears reliably in several samples grown in these conditions. Using a combination of X-ray diffraction (XRD) and AFM it is determined that the stripes are parallel to the $6 \mathrm{H}-\mathrm{SiC}$ substrate $<100>$ direct lattice vector.

Samples grown at a substrate temperature of $1200^{\circ} \mathrm{C}$ show oscillations of the specular RHEED spot brightness (Fig. 1(a)) which persist typically for the growth of at least $10 \mathrm{~nm}$ of $\mathrm{NbN}$, though $\mathrm{NbN}$ films over $30 \mathrm{~nm}$ in thickness have been grown exhibiting RHEED oscillations throughout the entirety of the film growth. We attribute these oscillations to a 2D Frank-van der Merwe (layer-by-layer) growth mode ${ }^{16}$. The thickness of material grown during a single RHEED oscillation, calculated using the measured film thickness, corresponds well with the $2.51 \AA$ interplanar lattice spacing of the film measured by XRD.

To our knowledge this is the first time a two-dimensional growth mode has been reported for $\mathrm{NbN}$ thin films grown by any method. As shown in Fig. 1(c), for films grown at 1200 ${ }^{\circ} \mathrm{C}$ the RHEED patterns are streaky with a $2 \times 2$ reconstruction pattern. AFM measurements of films grown at $1200{ }^{\circ} \mathrm{C}$ are consistent with layer-by-layer growth (Fig. 1(b)): the surfaces are atomically flat over large regions, with small features above and below the surface whose step heights measure approximately $2.5 \AA$, corresponding to the interplanar distance of ( $\left.\begin{array}{lll}1 & 1 & 1\end{array}\right)$ oriented $\delta$-NbN.

Growths were also attempted at substrate temperatures of $1300^{\circ} \mathrm{C}, 1500^{\circ} \mathrm{C}$, and $1700^{\circ} \mathrm{C}$. These films all exhibited RHEED patterns that were much dimmer and more diffuse than films grown at lower temperatures (Fig. 1c)), indicating poor crystalline quality. The surface of all samples grown at $1300{ }^{\circ} \mathrm{C}$ or higher had RMS roughness values of several nanometers and showed pits that were roughly as deep as the film thickness (Fig. 1(b)). A variety of nitrogen plasma conditions were attempted at these higher temperatures, but none resulted in films of higher crystal quality or smoother surface morphology.
The metallic and superconducting properties of $\mathrm{NbN}$ films depend sensitively on the crystal structure. The Nb-N crystal structure phase diagram is notably complex, containing at least 9 distinct $\mathrm{NbN}$ phases ${ }^{17-19}$. The rock-salt structure $\delta$-NbN phase possesses the highest superconducting critical temperature of all $\mathrm{NbN}$ phases and is therefore desired for many superconducting applications ${ }^{20}$. The primitive cubic $\mathrm{Nb}_{3} \mathrm{~N}_{3}$ and the tetragonal $\gamma-\mathrm{Nb}_{4} \mathrm{~N}_{3}$, both structurally similar to $\delta$-NbN, have also been shown to be superconductors with critical temperatures in excess of $10 \mathrm{~K}$, though for the structurally similar tetragonal $\mathrm{Nb}_{4} \mathrm{~N}_{5}$, no superconductivity has been observed for measured temperatures down to 1.77 $\mathrm{K}^{17}$. The hexagonal close packed $\beta-\mathrm{Nb}_{2} \mathrm{~N}$ has been reported to have a critical temperature as high as $8.6 \mathrm{~K}^{21}$, and the two related hexagonal phases $\delta$ ' $-\mathrm{NbN}$ and $\varepsilon-\mathrm{NbN}$, have been reported to not transition to the superconducting state above $1.77 \mathrm{~K}^{17,19}$, though recent reports claim higher transition temperatures for the $\varepsilon$ - $\mathrm{NbN}$ phase $\mathrm{p}^{22}$.
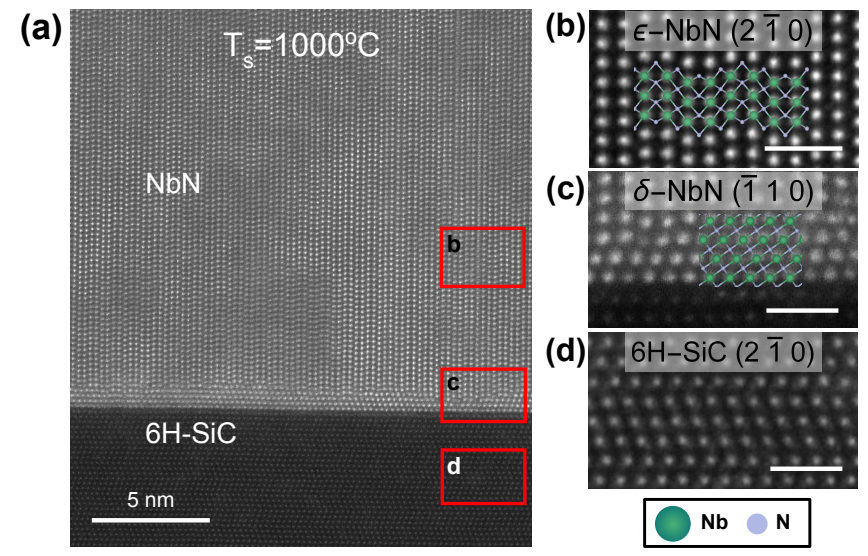

(e)
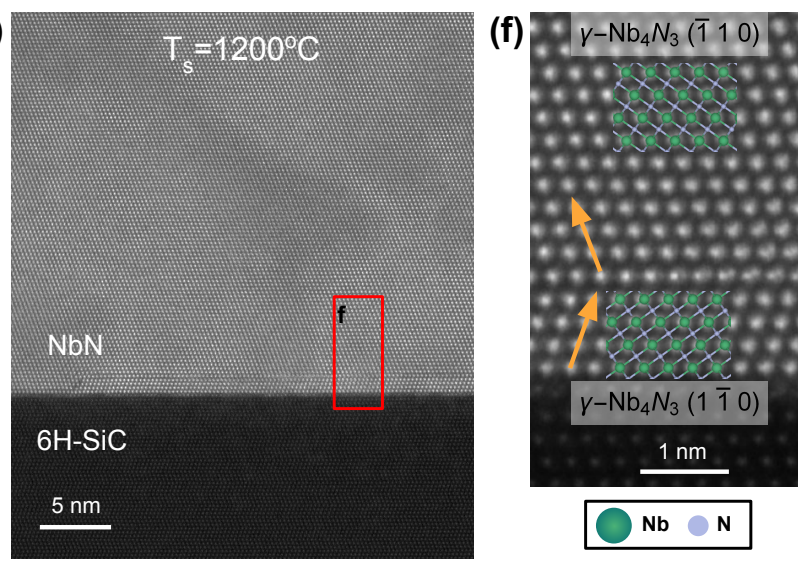

FIG. 2 : (a)-(d) HAADF-STEM image of an $\mathrm{NbN}$ film grown at 1000 ${ }^{\circ} \mathrm{C}$ shows a transition from cubic $\delta$-NbN to hexagonal $\varepsilon$-NbN a few unit cells away from the substrate. Scale bars in (b)-(d) correspond to $1 \mathrm{~nm}$. (e) \& (f) HAADF-STEM image of a NbN film grown at 1200 ${ }^{\circ} \mathrm{C}$ reveals a structure consistent with both the $\delta$-NbN phase and the closely related $\gamma-\mathrm{Nb}_{4} \mathrm{~N}_{3}$. Near the $6 \mathrm{H}-\mathrm{SiC} / \mathrm{NbN}$ interface are several layers of $\mathrm{NbN}$ that have a lattice orientation rotated $60^{\circ}$ about the growth axis relative to the orientation that is dominant in this image. 


\begin{tabular}{cccccccc}
\hline \hline $\mathrm{T}_{\mathrm{s}}\left({ }^{\circ} \mathrm{C}\right)$ & Phase $(\mathrm{s})$ & $\mathrm{T}_{\mathrm{c}}(\mathrm{K})$ & $\mathrm{AFM} \mathrm{R} \mathrm{RMS}_{\mathrm{Rm}}(\mathrm{nm}$ & $\mathrm{RRR}$ & $\rho @ 300 \mathrm{~K}(\mu \Omega \cdot \mathrm{cm})$ & $\mathrm{R}_{\mathrm{H}} @ 300 \mathrm{~K}\left(\mu \Omega \cdot \mathrm{cm}^{-1}\right)$ & $\mathrm{Nb}: \mathrm{N}$ ratio \\
\hline 800 & $\delta-\mathrm{NbN}$ & 10.55 & 0.61 & 0.45 & 207.03 & -0.0066 & $1.08 \pm 0.08$ \\
1000 & $\delta-\mathrm{NbN} / \varepsilon-\mathrm{NbN}$ & 15.5 & $0.79 / 0.21$ & 1.39 & 79.40 & 0.079 & $1.12 \pm 0.09$ \\
1200 & $\gamma-\mathrm{Nb}_{4} \mathrm{~N}_{3}$ & 6.98 & 0.11 & 4.68 & 37.18 & 0.0039 & $1.15 \pm 0.09$ \\
1300 & Unidentified & $<4$ & 6.53 & 1.3 & 56.17 & - & - \\
\hline \hline
\end{tabular}

TABLE I : Properties of $30 \mathrm{~nm} \mathrm{NbN}$ films grown on $6 \mathrm{H}-\mathrm{SiC}$.

We have used the combination of XRD, RHEED, and scanning transmission electron microscopy (STEM) to study the phase of the NbN films. The crystal phase(s) of each film is summarized in Table I. To determine the crystal structure of the $\mathrm{NbN}$ material grown at $1000{ }^{\circ} \mathrm{C}$, high angle annular dark field (HAADF-STEM) measurement was performed on a region with the striped surface morphology shown in Fig. 1(b). This measurement (Fig. 2(a)) indicates that the striped regions are composed predominantly of hexagonal $\varepsilon-\mathrm{NbN}$, with the growth-axis orientation $\varepsilon-\mathrm{NbN}\left[\begin{array}{lll}1 & 2 & 0\end{array}\right] / / 6 \mathrm{H}-\mathrm{SiC}\left[\begin{array}{lll}0 & 0 & 1\end{array}\right]$ and the in-plane orientation $\varepsilon-\mathrm{NbN}\left[\begin{array}{lll}0 & 0 & 1\end{array}\right] / / 6 \mathrm{H}-\mathrm{SiC}\left[\begin{array}{lll}1 & 0 & 0\end{array}\right]$. Therefore, the c-axis of the hexagonal $\mathrm{NbN}$ film is orthogonal to the c-axis of the substrate. Several layers of cubic $\delta$-NbN are observed near the $\mathrm{NbN} / 6 \mathrm{H}-\mathrm{SiC}$ interface, after which the film transitions to the hexagonal $\varepsilon-\mathrm{NbN}$ phase. This thin nucleation layer of $\delta$ - $\mathrm{NbN}$ was observed in all locations imaged. The regions in which the striped morphology did not appear were determined using XRD to correspond to the cubic $\delta$ $\mathrm{NbN}$ phase. It is unclear why only some regions of the film underwent a structural transition from $\delta$ - to $\varepsilon-\mathrm{NbN}$, and why it only occurs at this substrate temperature.

HAADF-STEM images of the $\mathrm{NbN}$ film grown at $1200{ }^{\circ} \mathrm{C}$ in Fig. 2(e)-(f) reveal a crystal structure consistent with cubic $\delta$-NbN. Several related phases, such as $\gamma-\mathrm{Nb}_{4} \mathrm{~N}_{3}$ in which the a and c lattice parameters differ by around $1 \%$, are difficult to distinguish from $\delta$-NbN based only on cross-sectional STEM images. However, XRD measurements provide evidence that the structure of the film is the tetragonal $\gamma-\mathrm{Nb}_{4} \mathrm{~N}_{3}$ phase, as discussed later. The first few layers above the substrate interface are observed to possess a lattice orientation rotated $60^{\circ}$ about the growth axis from the orientation that is dominant in this image. Given the 6-fold symmetry of $6 \mathrm{H}-\mathrm{SiC}$, both orientations of the $\mathrm{NbN}$ lattice are equivalent with respect to the substrate lattice.

Based on XRD 2 $\theta / \omega$ symmetric coupled scans (Fig. 3(a)) we confirm the presence of $\mathrm{NbN}$ phases with out-of-plane lattice parameters similar to that of the $6 \mathrm{H}-\mathrm{SiC}$ substrate for each film. However, given the large number of $\mathrm{NbN}$ phases with similar peak locations and the compositional variation possible within each phase ${ }^{20}$, these measurements of the out-ofplane film lattice parameter alone are insufficient to identify phases uniquely. To further characterize the crystal phase in each film we utilize asymmetric reciprocal space maps in the vicinity of reciprocal lattice points (RLP) of expected $\mathrm{NbN}$ phases. Fig. 3 shows that all three films exhibit a film peak

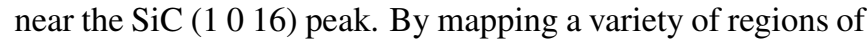
reciprocal space and detecting only peaks that correspond to $\delta$-NbN we conclude that the film grown at $800{ }^{\circ} \mathrm{C}$ is entirely $\delta$ - NbN. The $\delta$ - $\mathrm{NbN}$ phase grows with the growth-axis orien- tation $\delta$-NbN [llllll//SiC $\left.1 \begin{array}{lll}0 & 0 & 1\end{array}\right]$ and the in-plane orientations $\delta$-NbN [ $\left.\begin{array}{lll}1 & -1 & 0\end{array}\right] / / \mathrm{SiC}\left[\begin{array}{lll}1 & 0 & 0\end{array}\right]$ and $\delta$-NbN [ $\left.\begin{array}{lll}-1 & 1 & 0\end{array}\right] / / \mathrm{SiC}\left[\begin{array}{lll}1 & 0 & 0\end{array}\right]$. Due to the 6-fold symmetry of the substrate and the 3 -fold symmetry of the $\delta$ - $\mathrm{NbN}$ about the growth axis, two in-plane orientations of the $\delta$ - $\mathrm{NbN}$ lattice are possible, differing by a $60^{\circ}$ rotation about the growth axis. RHEED and XRD reciprocal space mapping (RSM) confirm the presence of both $\delta$ $\mathrm{NbN}$ orientations. Therefore, it is concluded that the $\delta$ - $\mathrm{NbN}$ films grown on $6 \mathrm{H}-\mathrm{SiC}$ are not single crystal but composed of cubic twin grains.
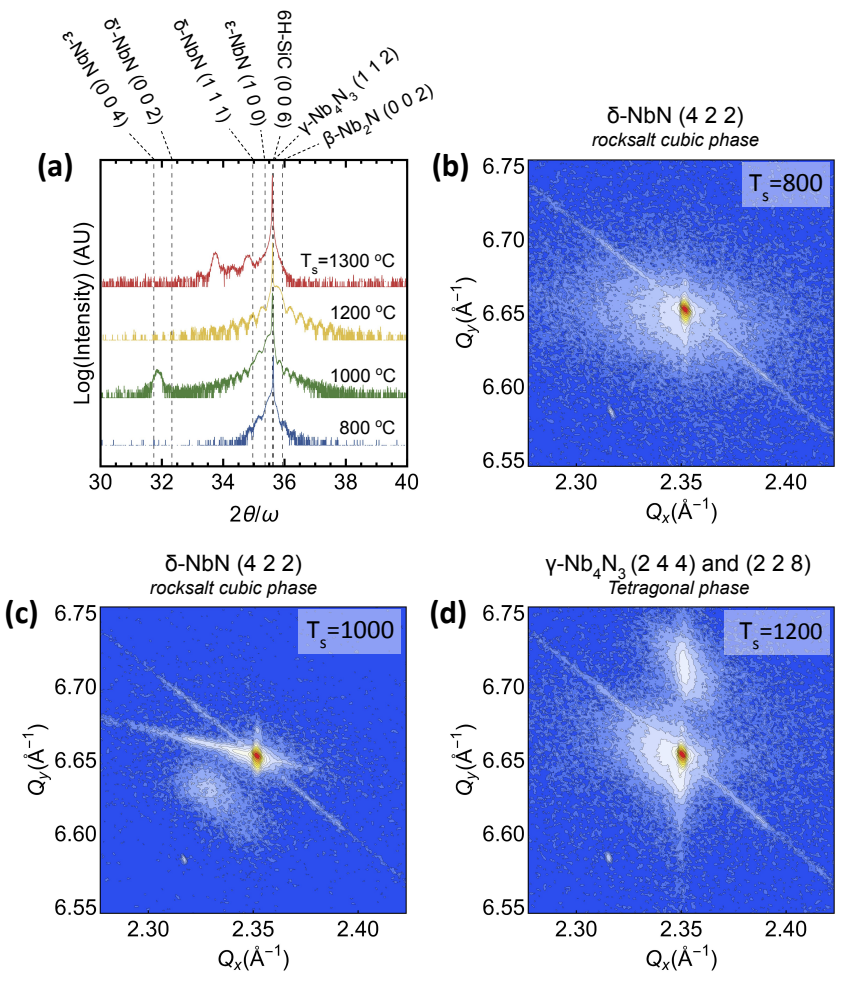

FIG. 3 : (a) Symmetric $2 \theta / \omega$ XRD scans of $30 \mathrm{~nm} \mathrm{NbN}$ thin films grown on $6 \mathrm{H}-\mathrm{SiC}$ substrate. (b)-(d) XRD reciprocal space maps of $\mathrm{NbN}$ films grown at (b) $800{ }^{\circ} \mathrm{C}$, (c) $1000{ }^{\circ} \mathrm{C}$, and (d) $1200{ }^{\circ} \mathrm{C}$. The region mapped is centered on the $6 \mathrm{H}-\mathrm{SiC}\left(\begin{array}{lll}1 & 0 & 16\end{array}\right)$ RLP. The expected epitaxial relationship of $\delta$-NbN on $6 \mathrm{H}$-SiC places the $\delta$-NbN (l $\left.\begin{array}{lll}4 & 2\end{array}\right)$ RLP near the 6H-SiC (1 0 16) RLP.

As seen in Fig. 3(d), the $\mathrm{NbN}$ film grown at $1200{ }^{\circ} \mathrm{C}$ exhibits a splitting of the NbN RLP such that there are 2 film peaks near the $6 \mathrm{H}-\mathrm{SiC}\left(\begin{array}{lll}1 & 0 & 16\end{array}\right)$ RLP. This splitting is evidence of tetragonal distortions of the rock-salt $\delta$ - NbN structure. This evidence in combination with the RHEED pattern allows us to conclude that the film possesses the $\gamma-\mathrm{Nb}_{4} \mathrm{~N}_{3}$ 
structure. A single-orientation $\gamma-\mathrm{Nb}_{4} \mathrm{~N}_{3}$ film would not exhibit two reciprocal lattice points at this location. However, for a film composed of tetragonal grains that are related by $120{ }^{\circ} \mathrm{C}$ rotation about the growth-axis, both the (2 4 4) and (2 2 8) reciprocal lattice points will be visible at this location. The grain structure can therefore be understood as the combined result of cubic twinning and tetragonal distortions of different lattice vectors in different regions of the film, producing 6 different orientations of the tetragonal structure, all of which are observed in the XRD analysis.

For both the film grown at $800{ }^{\circ} \mathrm{C}$ and $1200{ }^{\circ} \mathrm{C}$, the RSM (Fig. 3b-d) indicates that the in-plane lattice constant of the $\mathrm{NbN}$ is identical to that of the $6 \mathrm{H}-\mathrm{SiC}$, indicating that the films are strained. This strain requires a rhombohedral distortion to the cubic or tetragonal structures.

In addition to phase characterization, the $\mathrm{Nb}: \mathrm{N}$ ratio of samples grown at $800{ }^{\circ} \mathrm{C}, 1000{ }^{\circ} \mathrm{C}$, and $1200{ }^{\circ} \mathrm{C}$ was measured using Rutherford backscattering spectrometry (RBS) (Table I). All the films measured had $\mathrm{Nb}: \mathrm{N}$ ratios greater than 1 . The highest $\mathrm{Nb}: \mathrm{N}$ ratio measured was $1.15 \pm 0.09$ for the sample grown at $1200{ }^{\circ} \mathrm{C}$, with the variation in $\mathrm{Nb}: \mathrm{N}$ ratio between phases being within the uncertainty of the measurement.

To characterize the size and distribution of grains within the films, we performed lattice orientation mapping using scanning electron microscopy (SEM) based electron backscatter diffraction (EBSD). A $100 \mathrm{~nm}$ pure $\delta$-NbN film grown at $800{ }^{\circ} \mathrm{C}$ was used for EBSD analysis. The EBSD map (Fig. 4(a)) shows the interesting feature that the grains of different $\delta$ - $\mathrm{NbN}$ orientations form an alternating striped structure. In this film the grain width averages $548 \pm 52 \mathrm{~nm}$. AFM mapping (Fig 4(b)) of the same sample reveals parallel depressions with the same orientation and separation as the boundaries between grains observed in the EBSD map, and we therefore ascribe the depressions to the grain boundaries. A similar grain boundary is shown in Fig. 4(d) for a film grown at $1200^{\circ} \mathrm{C}$. In this image we observe triangular pyramidal surface features that rotate by $60^{\circ}$ on either side of the boundary, providing further evidence that the $\mathrm{NbN}$ lattice possesses different orientations on either side of the surface depression.

We hypothesized that the atomic steps of the $6 \mathrm{H}-\mathrm{SiC}$ substrate act as nucleation sites for the $\mathrm{NbN}$ film and therefore play a role in the formation of the observed grain structure. To test this hypothesis, we performed AFM mapping of a region of the substrate which was shadowed during growth of the film shown in Fig. 4(a)-(b). We observe atomic terraces with step-heights of $2.50 \pm 0.04 \AA$, which corresponds well with the height of one sixth of the $6 \mathrm{H}-\mathrm{SiC}$ c-axis unit cell (i.e. the height of a basal plane atomic layer). We find that the terrace widths average $407 \pm 38 \mathrm{~nm}$ and are not oriented along the same direction as the grain boundaries observed by EBSD and AFM. Therefore, this observation does not establish correlation between the substrate atomic terraces and the lamellar grain boundaries.

\section{B. Metallic conduction and Hall effect reversal}

The electrical properties of the films were characterized using temperature dependent Van der Pauw resistivity measurements. Low values of film resistivity are desirable for metallic (a)

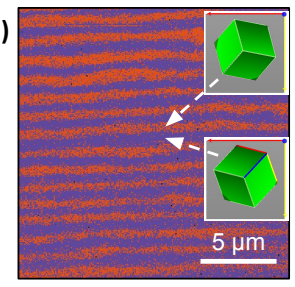

(c)
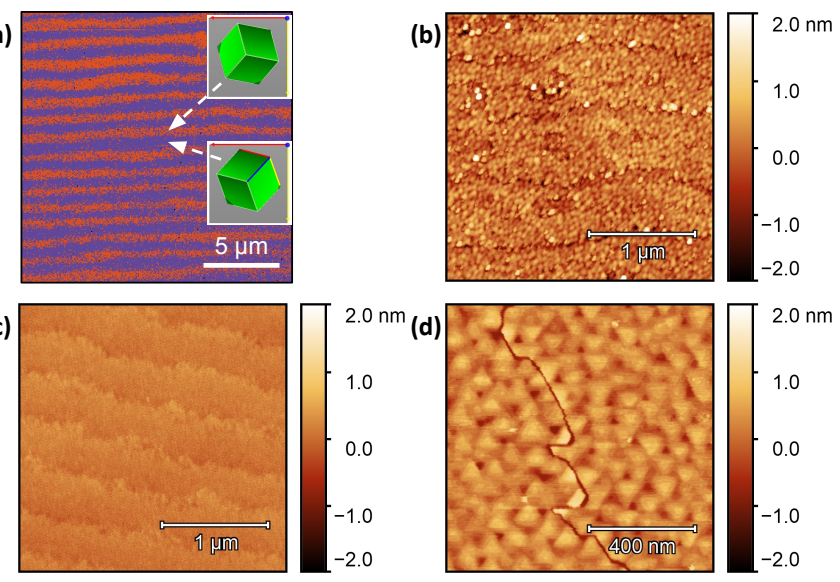

FIG. 4 : (a) The EBSD map of a $100 \mathrm{~nm} \mathrm{NbN}$ film reveals that the cubic twin grain boundaries form parallel stripes across the surface with width of approximately $550 \mathrm{~nm}$. (b) AFM surface mapping of the same $\mathrm{NbN}$ film shows a series of parallel depressions whose separation and orientation correspond with the grain boundaries observed in the EBSD map. (c) A region of the $6 \mathrm{H}-\mathrm{SiC}$ substrate that was shadowed during growth shows atomic terraces whose separation and orientation do not accurately match the grain boundaries orientation and separation. (d) AFM mapping of a $\mathrm{NbN}$ sample grown at $1200{ }^{\circ} \mathrm{C}$ at the site of a cubic twin grain boundary shows there is a depression at the site of the boundary approximately $1 \mathrm{~nm}$ deep.

applications of epitaxial $\mathrm{NbN}$ thin films ${ }^{23}$. We find that the resistivity of $\mathrm{NbN}$ samples grown at different temperatures varies over a large range as seen in Table I, with the minimum room-temperature resistivity of $37 \mu \Omega \cdot \mathrm{cm}$ observed for a film grown at $1200{ }^{\circ} \mathrm{C}$. The highest room temperature resistivity of $207 \mu \Omega \cdot \mathrm{cm}$ was observed in a film grown at $800{ }^{\circ} \mathrm{C}$. The lowest resistivity value previously reported for $\mathrm{NbN}$ thin films is $31.2 \mu \Omega \cdot \mathrm{cm}$ for a film $100 \mathrm{~nm}$ thick grown by reactive RF-magnetron sputtering ${ }^{5}$, indicating that further reduction of film resistivity for $\mathrm{MBE}$ grown $\mathrm{NbN}$ may still be possible.

The residual resistivity ratio (RRR), defined here as the ratio between the resistance at $300 \mathrm{~K}$ and the resistance at 20 $\mathrm{K}$ is used to compare the temperature dependence of resistivity of different films. As seen in Fig. 5(a) and Table I, the RRR varies directly with the growth temperature, starting at a value of 0.45 for the film grown at $800{ }^{\circ} \mathrm{C}$ and increasing to 4.7 for the film grown at $1200{ }^{\circ} \mathrm{C}$. The variation of RRR from values greater than one to values less than one has been reported previously in sputtered $\mathrm{NbN}$ films by Keskar et al., who found the RRR decreased as the partial pressure of nitrogen during sputtering increased at a constant substrate growth temperature ${ }^{24}$.

To further investigate the nature of conductivity of each film, Hall effect measurements of the $\mathrm{NbN}$ films were performed at temperatures ranging from $300 \mathrm{~K}$ to $20 \mathrm{~K}$ by sweeping the magnetic field from $-1 \mathrm{~T}$ to $+1 \mathrm{~T}$. The Hall voltage is confirmed to vary linearly with magnetic field over the range of magnetic fields tested. Fig. 5(c) shows the Hall coefficients as a function of temperature for the three $\mathrm{NbN}$ films grown at different substrate temperatures. For the films grown at 1000 ${ }^{\circ} \mathrm{C}$ and $1200{ }^{\circ} \mathrm{C}$ the Hall coefficient is positive at room temper- 

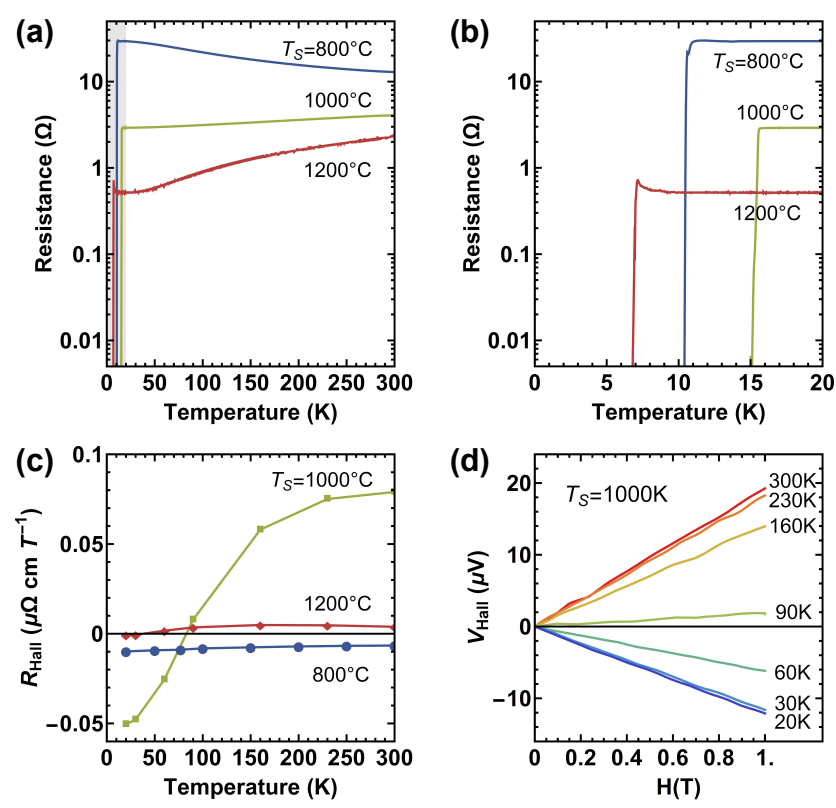

FIG. 5 : (a) Normal state resistance versus temperature of $\mathrm{NbN}$ thin films. (b) Resistance versus temperature in the range of the transition to the zero-resistance state. (c) Hall resistance versus temperature. (d) Hall voltage versus field for a single $\mathrm{NbN}$ film grown at $1000{ }^{\circ} \mathrm{C}$.

ature and becomes negative at temperatures less than approximately $85 \mathrm{~K}$ for the film grown at $1000{ }^{\circ} \mathrm{C}$ (Fig. $5(\mathrm{~d})$ ) and less than approximately $50 \mathrm{~K}$ for the film grown at $1200{ }^{\circ} \mathrm{C}$. For the sample grown at $800{ }^{\circ} \mathrm{C}$, the Hall coefficient is negative at all temperatures measured. Such a sign change in the Hall coefficient as a function of temperature has been observed in other single crystal metals with complex Fermi surfaces, such as indium ${ }^{25}$. Previous measurements of temperature dependent Hall coefficient of epitaxial $\mathrm{NbN}$ have observed negative Hall coefficients at all measurement temperatures ${ }^{26}$. To our knowledge this is the first report of the reversal of the Hall coefficient in $\mathrm{NbN}$ and the first report of a positive Hall coefficient at room temperature in $\mathrm{NbN}$. This observation indicates a richer range of $\mathrm{n}$ - and p-type regions of the Fermi surface of $\mathrm{NbN}$. It is necessary in the future to perform band structure measurements and 1st-principle calculations to explain this surprising observation, which is suggested as a future work.

\section{Superconductivity and Abrikosov vortex lattice}

The transition to the superconducting state is determined using Van der Pauw resistance measurements as a function of temperature (Fig 5(b)). The highest superconducting transition temperature observed was $15.5 \mathrm{~K}$ for a sample grown at $1000^{\circ} \mathrm{C}$. For comparison, the highest reported transition temperature for $\delta$-NbN thin films is approximately $17 \mathrm{~K}^{20}$. We note that the sample from this work with the highest transition temperature is a mixture of $\delta$ - $\mathrm{NbN}$ and $\varepsilon-\mathrm{NbN}$, and therefore the superconducting critical temperature of different regions of the film likely differs. The $\mathrm{NbN}$ samples grown at $800^{\circ} \mathrm{C}$, which were determined to be purely $\delta$-NbN, exhibited a lower critical temperature of $10.6 \mathrm{~K}$. We therefore conclude that the growth of a phase pure $\delta$ - $\mathrm{NbN}$ film is neither necessary nor sufficient to achieve a film with high superconducting critical temperature. Samples grown at $1300^{\circ} \mathrm{C}$ show no transition to the superconducting state above $4 \mathrm{~K}$.

To further investigate properties of the superconducting state of the NbN films, scanning tunneling microscopy (STM) measurements were performed at $4.7 \mathrm{~K}$ for a single $\mathrm{NbN}$ film grown at $1200{ }^{\circ} \mathrm{C}$. Differential conductance measurements, shown in Fig. 6(a), are used to characterize the superconducting energy gap of the film. The superconducting energy gap is determined by modelling the differential conductance based on the BCS density of states ${ }^{27}$, yielding an energy gap of $\Delta=2.55 \pm 0.01 \mathrm{meV}$. Fig. 6(b) shows the surface topography near an atomic step and Fig. 6(c) shows a waterfall plot of the differential conductance across the region imaged in Fig 6(b). We observe that the superconducting energy gap is uniform across the sample surface, including across an atomic step and a surface defect that appears in the topography scan.
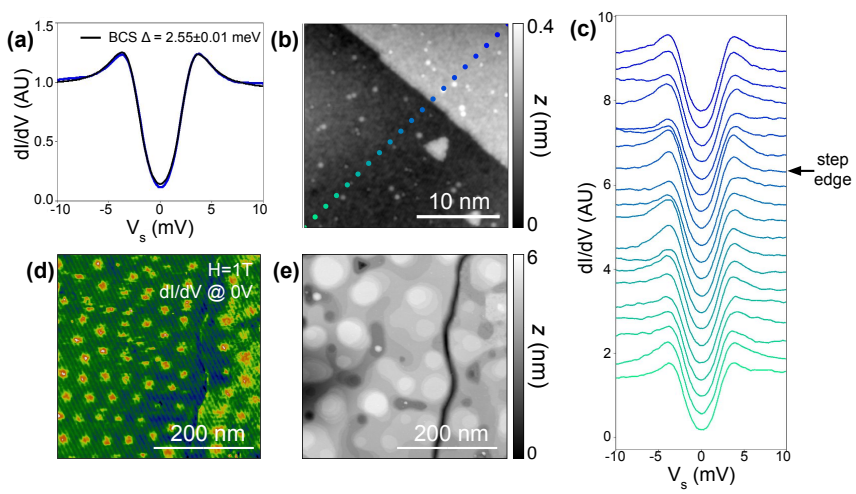

FIG. 6 : (a)-(d) Scanning tunneling microscope differential conductance measurements at $4.7 \mathrm{~K}$ of a NbN film grown at $\mathrm{T}_{\mathrm{s}}=1200^{\circ} \mathrm{C}$ : (a) differential conductance versus voltage is well fit using the BCS density of states yielding the energy gap of the NbN film. (b) Surface topography map and (c) differential conductance waterfall plot shows a highly uniform superconducting energy gap across the sample surface. (d) Differential conductance map and (e) surface topography map in the presence of a $1 \mathrm{~T}$ magnetic field shows an Abrikosov vortex lattice that is distorted in the vicinity of a cubic twinning grain boundary. Sample bias was $0.1 \mathrm{~V}$ and constant current was $10 \mathrm{pA}$ for both (b) and (e), and modulation voltage was $0.5 \mathrm{mV}$ peak-topeak for (a), (c), and (d).

Fig. 6(d) shows a differential conductance map at $0 \mathrm{~V}$ in the presence of a magnetic field of $1 \mathrm{~T}$, enabling us to image the presence of Abrikosov vortices. As is apparent in Fig. 6(d) the Abrikosov vortices form a triangular lattice, an indication of the lack of strong pinning of vortices at the sites of material defects. Previous reports of STM analysis of epitaxial NbN thin films did not observe the formation of an ordered vortex lattice ${ }^{10}$. Fig 6(e) shows the surface topography of the region imaged in Fig 6(d), revealing a depression in the surface that we attribute to a grain boundary between cubic twin grains due to the similarity of the feature to that shown in Fig. 4(d). It is apparent in Fig. 6(d) that the Abrikosov vortex lattice near the cubic twin domain boundary is distorted, indicating that the domain boundary affects the local superconducting state. 


\section{DISCUSSION}

We have shown in this study that the growth mode, morphology, phase, and electronic properties of $\mathrm{NbN}$ thin films grown by $\mathrm{MBE}$ are strongly influenced by substrate growth temperature. With this in mind we note that achieving uniform and controlled film properties is complicated by the fact for a constant substrate thermocouple temperature the film surface temperature increases during growth as the $\mathrm{NbN}$ film itself increases infrared absorption from the substrate heater ${ }^{15}$.

Previous work on the $\mathrm{Nb}-\mathrm{N}$ phase diagram has predicted that the $\delta$ - $\mathrm{NbN}$ phase is thermodynamically stable only at temperatures exceeding $1300{ }^{\circ} \mathrm{C}^{28}$. It is therefore interesting to note that in the highly non-equilibrium conditions of MBE, films of pure $\delta$ - $\mathrm{NbN}$ phase appear at substrate temperatures much below this, and that no $\delta$-NbN films have been grown above $1300{ }^{\circ} \mathrm{C}$ despite repeated attempts with a variety of nitrogen and $\mathrm{Nb}$ flux ratios and growth rates.

The reversal of the Hall coefficient as a function of temperature in a $\mathrm{NbN}$ thin film is a new and surprising observation. Films grown at $800{ }^{\circ} \mathrm{C}$ showed negative Hall coefficient at all temperatures, and those grown at $1000{ }^{\circ} \mathrm{C}$ and $1200{ }^{\circ} \mathrm{C}$ showed positive Hall coefficient at room temperature and negative Hall coefficient at temperatures below $85 \mathrm{~K}$ and $50 \mathrm{~K}$ respectively. We have demonstrated that films grown at different temperatures differ in growth mode, grain size, crystal phase, and potentially $\mathrm{Nb}: \mathrm{N}$ ratio, and therefore are currently unable to simply attribute differences in electronic properties between the films to any of the other observed differences between the films. A combination of band structure measurement and theory of the magnetoconductivity and Hall effect in single crystal $\mathrm{NbN}$ is needed to explain the surprising Hall effect reversal. The observation of an ordered Abrikosov vortex lattices indicates the feasibility of high-quality epi-NbN for flux-based classical and quantum computation applications.

Although layer-by-layer growth of $\mathrm{NbN}$ has been achieved, removing all grain boundaries from the films has not, due to the symmetry mismatch between the substrate and the film. The result is a film composed of single orientation grains approximately $500 \mathrm{~nm}$ wide and at least $100 \mu \mathrm{m}$ long. Initial evidence from STM measurements indicates that these grain boundaries do affect the superconducting state. The importance of these boundaries will ultimately depend on the intended application of the film. We note that for some applications the grains are large enough and the boundaries are easy enough to locate that it should be possible to fabricate electronic devices on individual single orientation superconducting regions.

\section{ACKNOWLEDGMENTS}

This work used the CNF, CCMR and CESI Shared Facilities partly supported by the NSF NNCI program (ECCS1542081), MRSEC program (DMR-1719875) and MRI DMR-1338010 and Kavli Institute at Cornell (KIC). We acknowledge funding support from the Office of Naval Research, monitored by P. Maki under award numbers N0001420-1-2176 and N00014-17-1-2414.

\section{AUTHOR CONTRIBUTIONS}

J. Wright prepared the films and performed the XRD, AFM, EBSD, and temperature dependent transport measurements. C. Chang and D. Muller performed the STEM measurements. D. Waters, F. Lüpke, and R. Feenstra performed the STM measurements. L. Raymond assisted with XRD measurements. R. Koscica assisted with Hall effect measurements. G. Khalsa, D. Jena, and H.G. Xing coordinated the work and provided experimental and theoretical guidance. J. Wright and D. Jena wrote the paper.

\section{COMPETING INTERESTS}

The authors declare that they have no conflict of interest.

\section{DATA AVAILABILITY}

The data that support the findings of this study are available from the corresponding author upon reasonable request.

\section{REFERENCES}

${ }^{1}$ G. Aschermann, E. Friederich, E. Justi, and J. Kramer, "Supraleitfahige Verbindungen mit extrem hohen Springtemperaturen," Physik Z. 42, 349 (1941).

${ }^{2}$ Y. Nakamura, H. Terai, K. Inomata, T. Yamamoto, W. Qiu, and Z. Wang, "Superconducting qubits consisting of epitaxially grown NbN/AlN/NbN Josephson junctions," Applied Physics Letters 99, 212502 (2011).

${ }^{3}$ G. N. Gol'tsman, O. Okunev, G. Chulkova, A. Lipatov, A. Semenov, K. Smirnov, B. Voronov, A. Dzardanov, C. Williams, and R. Sobolewski, "Picosecond superconducting single-photon optical detector," Applied Physics Letters 79, 705-707 (2001).

${ }^{4} \mathrm{H}$. Terai and Z. Wang, "9 K operation of RSFQ logic cells fabricated by $\mathrm{NbN}$ integrated circuit technology," in IEEE Transactions on Applied Superconductivity, Vol. 11 (2001) pp. 525-529.

${ }^{5}$ A. Shoji, S. Kiryu, and S. Kohjiro, "Superconducting properties and normal-state resistivity of single-crystal $\mathrm{NbN}$ films prepared by a reactive rf-magnetron sputtering method," Applied Physics Letters 60, 1624-1626 (1992).

${ }^{6}$ K. Senapati, N. K. Pandey, R. Nagar, and R. C. Budhani, "Normal-state transport and vortex dynamics in thin films of two structural polymorphs of superconducting NbN," Physical Review B - Condensed Matter and Materials Physics 74, 104514 (2006).

${ }^{7}$ M. Ziegler, L. Fritzsch, J. Day, S. Linzen, S. Anders, J. Toussaint, and H. G. Meyer, "Superconducting niobium nitride thin films deposited by metal organic plasma-enhanced atomic layer deposition," Superconductor Science and Technology 26, 025008 (2013).

${ }^{8}$ D. Hazra, N. Tsavdaris, S. Jebari, A. Grimm, F. Blanchet, F. Mercier, E. Blanquet, C. Chapelier, and M. Hofheinz, "Superconducting properties of very high quality $\mathrm{NbN}$ thin films grown by high temperature chemical vapor deposition," Superconductor Science and Technology 29, 105011 (2016).

${ }^{9}$ D. S. Katzer, N. Nepal, M. T. Hardy, B. P. Downey, D. F. Storm, E. N. Jin, R. Yan, G. Khalsa, J. Wright, A. C. Lang, T. A. Growden, V. Gokhale, V. D. Wheeler, A. R. Kramer, J. E. Yater, H. G. Xing, D. Jena, and D. J. Meyer, "Molecular Beam Epitaxy of Transition Metal Nitrides for Superconducting Device Applications," Physica Status Solidi (A) Applications and Materials Science 217, 1900675 (2020).

${ }^{10}$ G. Y. Wang, Z. Zhu, X. Y. Yang, L. Dong, H. Y. Ma, H. H. Sun, A. M. Li, D. D. Guan, D. Qian, C. Liu, Y. Y. Li, and J. F. Jia, "Atomically flat superconducting NbN thin films grown on SrTiO3 (111) by plasma-assisted MBE," APL Materials 5, 126107 (2017).

${ }^{11}$ R. Simmonds, K. Lang, D. Hite, S. Nam, D. Pappas, and J. Martinis, "Decoherence in Josephson Phase Qubits from Junction Resonators,' Physical Review Letters 93, 077003 (2004).

${ }^{12}$ R. Yan, G. Khalsa, S. Vishwanath, Y. Han, J. Wright, S. Rouvimov, D. S. Katzer, N. Nepal, B. P. Downey, D. A. Muller, H. G. Xing, D. J. Meyer, and 
D. Jena, "GaN/NbN epitaxial semiconductor/superconductor heterostructures," Nature 555, 183-189 (2018).

${ }^{13}$ D. Jena, R. Page, J. Casamento, P. Dang, J. Singhal, Z. Zhang, J. Wright, G. Khalsa, Y. Cho, and H. G. Xing, "The new nitrides: layered, ferroelectric, magnetic, metallic and superconducting nitrides to boost the GaN photonics and electronics eco-system," Japanese Journal of Applied Physics 58, SC0801 (2019).

${ }^{14} \mathrm{H}$. Okumura, Formation Mechanism of Extended Defects in AlN Grown on $\mathrm{SiC}\{0001\}$ and Their Reduction by Initial Growth Control, Ph.D. thesis, Kyoto University (2012).

${ }^{15}$ D. Scott Katzer, M. T. Hardy, N. Nepal, B. P. Downey, E. N. Jin, and D. J. Meyer, "Growth-induced temperature changes during transition metal nitride epitaxy on transparent SiC substrates," Journal of Vacuum Science \& Technology B 38, 032204 (2020).

${ }^{16} \mathrm{~W}$. Braun, Applied RHEED (Springer, Berlin, 1999).

${ }^{17}$ G.-I. Oya and Y. Onodera, "Transition temperatures and crystal structures of single-crystal and polycrystalline $\mathrm{NbN}_{\mathrm{X}}$ films," Journal of Applied Physics 45, 1389-1397 (1974).

${ }^{18}$ R. E. Treece, M. S. Osofsky, E. F. Skelton, S. B. Qadri, J. S. Horwitz, and D. B. Chrisey, "New phase of superconducting $\mathrm{NbN}$ stabilized by heteroepitaxial film growth," Physical Review B 51, 9356-9359 (1995).

${ }^{19}$ K. R. Babu and G. Y. Guo, "Electron-phonon coupling, superconductivity, and nontrivial band topology in NbN polytypes," Physical Review B 99 (2019), 10.1103/PhysRevB.99.104508, arXiv:1808.05073.

${ }^{20} \mathrm{~W}$. Lengauer, "Characterization of nitrogen distribution profiles in FCC transition metal nitrides by means of $\mathrm{T}_{\mathrm{C}}$ measurements," Surface and Interface Analysis 15, 377-382 (1990).

${ }^{21}$ J. Gavaler, J. Hulm, M. Janocko, and C. Jones, "Preparation and Superconducting Properties of thin films of transition metal interstitial compounds,"
J Vacuum Science \& Technology 6, 177-180 (1969).

${ }^{22}$ Y. Zou, X. Qi, C. Zhang, S. Ma, W. Zhang, Y. Li, T. Chen, X. Wang, Z. Chen, D. Welch, P. Zhu, B. Liu, Q. Li, T. Cui, and B. Li, "Discovery of superconductivity in hard hexagonal $\varepsilon$-NbN," Scientific Reports 6 (2016), $10.1038 /$ srep22330.

${ }^{23}$ J. Miller, J. Wright, H. G. Xing, and D. Jena, "All-Epitaxial Bulk Acoustic Wave Resonators," physica status solidi (a) (2020), 10.1002/pssa.201900786.

${ }^{24}$ K. S. Keskar, T. Yamashita, and Y. Onodera, "Superconducting Transition Temperatures of R. F. Sputtered NbN Films," Japanese Journal of Applied Physics 10, 370-374 (1971).

${ }^{25}$ E. J. Ozimek, R. G. Leisure, and D. K. Hsu, "Reversal of the Hall field in indium," Physics Letters A 66, 413-415 (1978).

${ }^{26}$ M. Chand, A. Mishra, Y. M. Xiong, A. Kamlapure, S. P. Chockalingam, J. Jesudasan, V. Bagwe, M. Mondal, P. W. Adams, V. Tripathi, and P. Raychaudhuri, "Temperature dependence of resistivity and Hall coefficient in strongly disordered NbN thin films," Physical Review B - Condensed Matter and Materials Physics 80, 134514 (2009).

${ }^{27}$ F. Lüpke, D. Waters, S. C. de la Barrera, M. Widom, D. G. Mandrus, J. Yan, R. M. Feenstra, and B. M. Hunt, "Proximity-induced superconducting gap in the quantum spin Hall edge state of monolayer WTe2," (2020), arXiv:1903.00493.

${ }^{28}$ E. M. Levin, A. E. MacHale, H. F. MacMurdie, and C. R. Robbins, Phase diagrams for ceramists. Vol. 10: Borides, Carbides, and Nitrides (The American Ceramic Society, Columbus, Ohio, 1994). 